\title{
AN EFFECT OF WASHING ON DURABILITY OF HYDROPHOBIC FINISHES OF MEMBRANE LAMINATES
}

\author{
Mateusz Kowalski, Renata Salerno-Kochan \\ Cracow University of Economics - Faculty of Commodity Science and Product Management, Sienkiewicza 4, \\ 30-033 Cracow, Poland, kowalskm@uek.krakow.pl; salernor@uek.krakow.pl
}

\begin{abstract}
:
The aim of the study is to show how the washing process, while considering the type of washing agent and number of wash cycles, determines the durability of hydrophobic finishes of laminated fabrics. The samples of material were subject to multiple wash cycles in clear water and with the use of two types of washing agents, namely, an ordinary washing powder and a detergent recommended by manufacturers of functional clothing. The washed materials were subject to spray test and its waterproofing and degree of surface wetting were evaluated. The tests have shown that waterproofing of membrane laminates with DWR (durable water repellent) coating become deteriorated during washing treatment and the use of special detergent does not guaranty the maintenance of better coating properties than an ordinary washing agent.
\end{abstract}

\section{Keywords:}

hydrophobic finishes, fabric washing, water repellency, membrane laminates

\section{Introduction}

The main function of outer layer of clothing made of laminated materials is to protect against adverse weather factors, among which precipitation is the most invasive one. Rain and melted snow can cause wetting of the outer layer and in consequence may lead to water penetration into deeper layers. Wet clothing can lead to disruption of body thermal balance, thus causing significant deterioration of user comfort [14]. To prevent it, the outer layers of clothing should be resistant to water (waterproofing) that includes two basic material properties, namely, water tightness and wetting resistance (hydrophobic abilities/water repellency) [12].

Water tightness is reached, among other things, by using membrane materials, which prevent the penetration of water into the inner layers of clothing and shed rain droplets or melted snow on its surface, while water repellency is most often an effect of shell materials of appropriate dense structure based on the hydrophobic fibers. The manufacturers more often use also hydrophobic finishes that form a waterproof coating on the material surface that helps to strengthen the product's barrier to water $[7,11,12-13,15]$. These finishes are called as DWR (durable water repellent) coatings. Their main goal is to create a thin membrane on its surface preventing the spilling of liquid droplets and wetting the inner clothing layers [16]. The mechanism of action of such coating, illustrating the behavior of water droplets on its surface, is presented in Figure 1.

It is possible to achieve hydrophobicity by enabling liquid droplets of the highest possible contact angle to be formed that promotes its rundown without causing the wetting of material by using coatings of a surface energy lower than the surface tension of water. Any liquid of lower surface tension can spill over the surface of solid body of higher surface tension, while an inverse phenomenon is impossible. A solid body (textile material) of low surface energy gained with a DWR finish is comprised mainly of non-polar groups, while water is a polar solvent; thus when these two bodies contact each other, a repulsion phenomenon occurs. This is why water dropping on the material forms no wet continuous layer on it but "transforms" into individual droplets (similar to pearls) that can be easily removed from the surface of a material without leaving any wet traces [10].

Wettability of a material depends on the contact angle. The contact angle $(\theta)$ is the angle between the tangent to the test droplet outer surface and the solid surface where the liquid is present at interface of three phases: solid (S), liquid (L), and vapor (gaseous) (V). The relationship between these phases under equilibrium conditions is expressed by the Young equation, which is the basis for describing all wetting phenomena $[2,9]$ :

$$
\gamma_{\mathrm{SV}}-\gamma_{\mathrm{SL}}=\gamma_{\mathrm{LV}} \cdot \cos \theta
$$

where $\gamma_{\mathrm{sV}}$ is the surface tension in a solid-gas system (here: air), $\gamma_{S L}$ is the surface tension in a solid-liquid system, $\gamma_{L V}$ is the surface tension in a liquid-gas system, $\theta$ is the wetting contact angle. hydrophilic surface

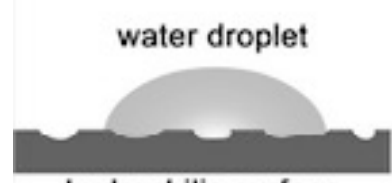

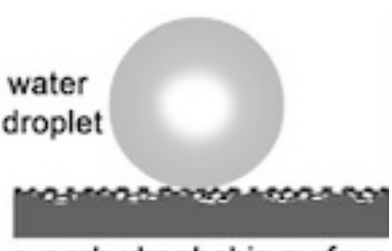

superhydrophobic surface
Figure 1. The mechanism of hydrophobic coatings activity [17] 
The situation described by the Young equation is shown in Figure 2.

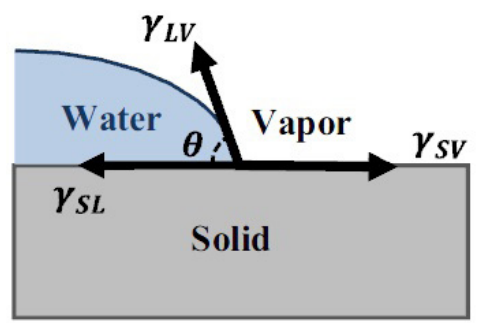

Figure 2. The behavior of droplets of liquid on the three-phase border [8]

The value of contact angle indicates water-related properties of the surface of materials. If the contact angle is acute $(\theta<$ $90^{\circ}$ ), the surface of material undergoes complete wetting in contact with water. This refers to high-energy surfaces, the socalled hydrophilic (high wettable). Greater contact angles $90^{\circ}<$ $\theta<150^{\circ}$ cause that the surface is hydrophobic (low wettable). The surfaces that have contact angles greater than $150^{\circ}$ are termed superhydrophobic (Fig. 1). Such properties are the characteristic of materials used for manufacturing self-cleaning textiles. However, if the contact angle is close to $180^{\circ}$, such surface is water nonwettable (e.g., lotus leaf: $\theta \approx 170^{\circ}$ ) $[3,8]$.

Textile raw materials used in the manufacture of clothing typically feature surface energy higher than water surface tension; thus in majority of cases, they are susceptible to wetting or show slight hydrophobic abilities. To improve hydrophobic properties of materials, appropriate chemical modifications of its surface are used to get water contact angles of about $120^{\circ}$. At present, fluorocarbon polymers are chemical compounds most often used to impart hydrophobic properties to products [5]. The widespread use of fluorocarbons results from its very low surface energy and also strong hydrophobic properties. The efficiency of its hydrophobic action is connected with the structure of chemical bonds in molecules. The connections between atoms of carbon and fluorine are very short, thus stronger, so fluoropolymer coatings are resistant to wetting by water. An example of polymer of such properties is polytetrafluoroethylene (PTFE) for which the contact angle does not exceed $120^{\circ}[3-4,9]$.
The durability of fluorochemical finishes is generally quite sufficient, although it depends to large extent on the ultimate application of products. This is highly affected by weather conditions such as heavy rain, solar irradiance, humidity, as well as factors related to product use, for example, material exposure to multiple abrasions, pressure, and clothing preservation processes [4].

Clothing maintenance is one of the key factors that influence the original properties of the product. It is necessary to use it because when using, the products become soiled and retain contaminants of various origin, including dust, fat, sweat, etc., that may deteriorate clothing functionality. On the other hand, improper maintenance, for example, using improper washing agent, may lead to damage to textile structure and for coated materials to coating damage, thus to deterioration or even loss of waterproofing qualities. The manufacturers warn about it and recommend their customers to purchase specialized dedicated washing agents to maintain functional products.

Bearing the above in mind, an attempt was made to show how the washing process, while considering the type of washing agent, determines the durability of hydrophobic finishes of laminated fabrics. The hypothesis was posted that the washing process has an adverse effect on waterproofing properties of laminated fabrics, while an appropriate detergent selection allows these qualities to be maintained for a longer period of use.

\section{Experimental}

\subsection{Materials}

This study was carried out on four two-layer membrane laminates that, according to information provided by manufacturers, were used for trekking and ski jackets. All materials were finished with DWR coating. Data obtained from the producer and own research (Table 1) indicates that differences between laminates under analyzes refer to the membrane type, properties, as well the structure of outer fabrics.

Table 1. Characteristics of two-layer laminates

\begin{tabular}{|c|c|c|c|c|}
\hline \multirow{2}{*}{ CHARACTERISTICS } & \multicolumn{4}{|c|}{ SAMPLE } \\
\hline & $\mathbf{A}$ & B & C & D \\
\hline Breathability*,$g / m^{2} \times 24 h$ & 3,000 & 3,000 & 2,000 & 3,000 \\
\hline Waterproofness of membrane ${ }^{*}, \mathrm{~mm} / \mathrm{m}^{2}$ & 5,000 & 5,000 & 3,000 & 5,000 \\
\hline Mass per unit area, $\mathbf{g} / \mathrm{m}^{2}$ & 165 & 170 & 175 & 175 \\
\hline Thickness, mm & 0.36 & 0.31 & 0.28 & 0.28 \\
\hline Color of the fabric & Black & Red & Blue & Green \\
\hline Fiber composition of the outside fabric & PES & PES & PES & PES \\
\hline Kind of weave of the outside fabric & Twill $\frac{2}{1}(Z)$ & Twill $\frac{2}{1}(Z)$ & Twill $\frac{2}{1}(Z)$ & Twill $\frac{2}{1}(\mathrm{~S})$ \\
\hline Liner density, no. of yarns $/ 1 \mathrm{~cm}^{2}$ & 90 & 90 & 105 & 88 \\
\hline Color of membrane & Grey-white & Milk-white & Transparent & White \\
\hline Membrane composition & PU & PVDF & PU & PU \\
\hline
\end{tabular}

* Approximate parameter values based on the manufacturer data 
The outer laminate fabrics had the same composition $(100 \%$ PES) and twill weave. Among the analyzed materials, sample C differed mostly from the other samples because of its lower water tightness and the highest linear density of outer fabric. For sample $B$, the presence of another type of membrane based on polyvinylidene fluoride was found, while other samples contained polyurethane membranes. Two types of washing agents were used during tests, including widely used washing powder of the leading manufacturer in the laundry detergent market (DETERGENT I) and a liquid detergent designed for washing sportswear "in particular, breathable, waterproof, wind-tight, membrane, cotton and other difficult to care fabrics," recommended by a manufacturer of sports clothing and accessories (DETERGENT II).

To evaluate the effect of type of detergent used on the hydrophobic finish, the samples washed in two types of washing agents were compared with the behavior of samples washed with water alone without the use of detergents, while maintaining the same preservation conditions, that is, number of wash cycles performed, water volume, and temperature as well as washing, rinsing, and drying times.

\subsection{Methods}

The water-repellent efficacy of finishes was determined according to the AATCC Test Method 22-2005 (the so-called spray test). The durability of hydrophobic finish was assessed organoleptically by using photographic reference standards (Fig. 3) to determine the degree of wetting for outer textile expressed on a scale 100-0.
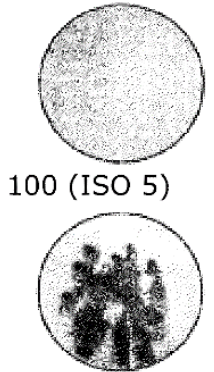

70 (ISO 2)
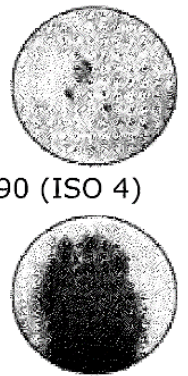

50 (ISO 1)
90 (ISO 4)

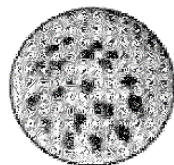

80 (ISO 3)

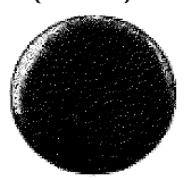

0
Figure 3. Photographic reference standards used for assessing the degree of sample wetting [1]

An analysis of water droplet size and behavior on the surface of sprayed material was carried out based on the methodology of determining the resistance of textile products to rain according to the Bundesmann rain-shower test (Fig. 4) [6]. The assessment was based on a 5-point scale of photographic reference standards, where grade 5 indicates a high finish durability (small, spherical droplets rapidly running down), while grade 1 indicates a low-quality finish (sample wets all over its surface).

The washing procedure was developed according to the instructions for domestic washing consistent with EN ISO 12138. The amount of detergent added each time to washing bath regarding mass or volume was adjusted to recommendations specified on washing agent packages. The washing temperature was selected based on the washing instructions placed on labels of multilayer laminated clothing by their manufacturers $\left(30^{\circ} \mathrm{C}\right)$. To ensure washing conditions closest to those of domestic washing, rinsing was carried out in water at $15 \pm 1^{\circ} \mathrm{C}$. After washing, the samples were dried for $24 \mathrm{~h}$.

\section{Results and discussion}

According to the methodology used, the durability of hydrophobic finish was assessed organoleptically by paying attention on water droplet behavior on the fabric surface and evaluating the degree of outer fabric wetting. Evaluation of the durability of DWR coatings, made after spray test, was developed in the form of diagrams, which are presented in Figure 5.

An analysis of control samples (unwashed) undergoing testing as well as water droplet behavior on their surface indicated that all materials have very good (sample A and B) or good (sample $C$ and $D$ ) degree of waterproofing. Small differences in the assessment of materials under investigation were related to water droplet size and behavior on the surface. Water droplets on the surface of samples A and B were clearly smaller and easier to shed, while they formed larger aggregations for samples $C$ and D. No wetting traces were observed in all samples. No correlation was found between structural parameters of outer fabrics (linear density, mass per square meter, thickness) and waterproof properties of tested laminates.

After the first wash cycle performed with an ordinary washing powder (DETERGENT I), some unfavorable effects occurred in the form of wet stains, especially visible on the surface of sample $A$, and also, to a lesser degree, on the surface of samples B, $C$, and $D$. In sample $C$, the wetting traces were located at a fold made when the laminate was previously folded. Similar phenomenon was observed also in sample D (Fig. 6) washed with a special liquid detergent (DETERGENT II). This suggests that hydrophobic coatings are very vulnerable to bending that occurs during normal use and one should expect decreased waterproofing properties at spots exposed to bending. For

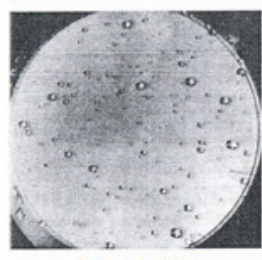

Grade 5

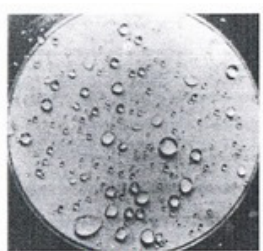

Grade 4

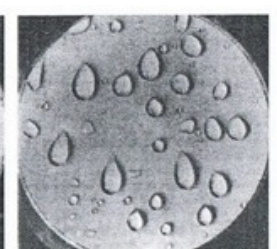

Grade 3

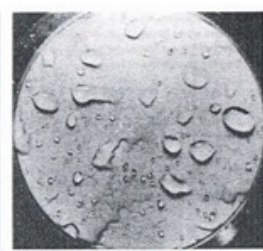

Grade 2

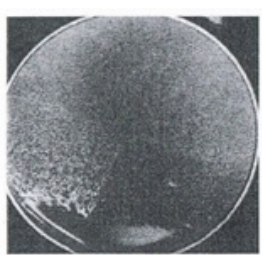

Grade 1

Figure 4. Photographic reference standards used for assessing the water repellency of fabrics by the Bundesmann rain-shower test 


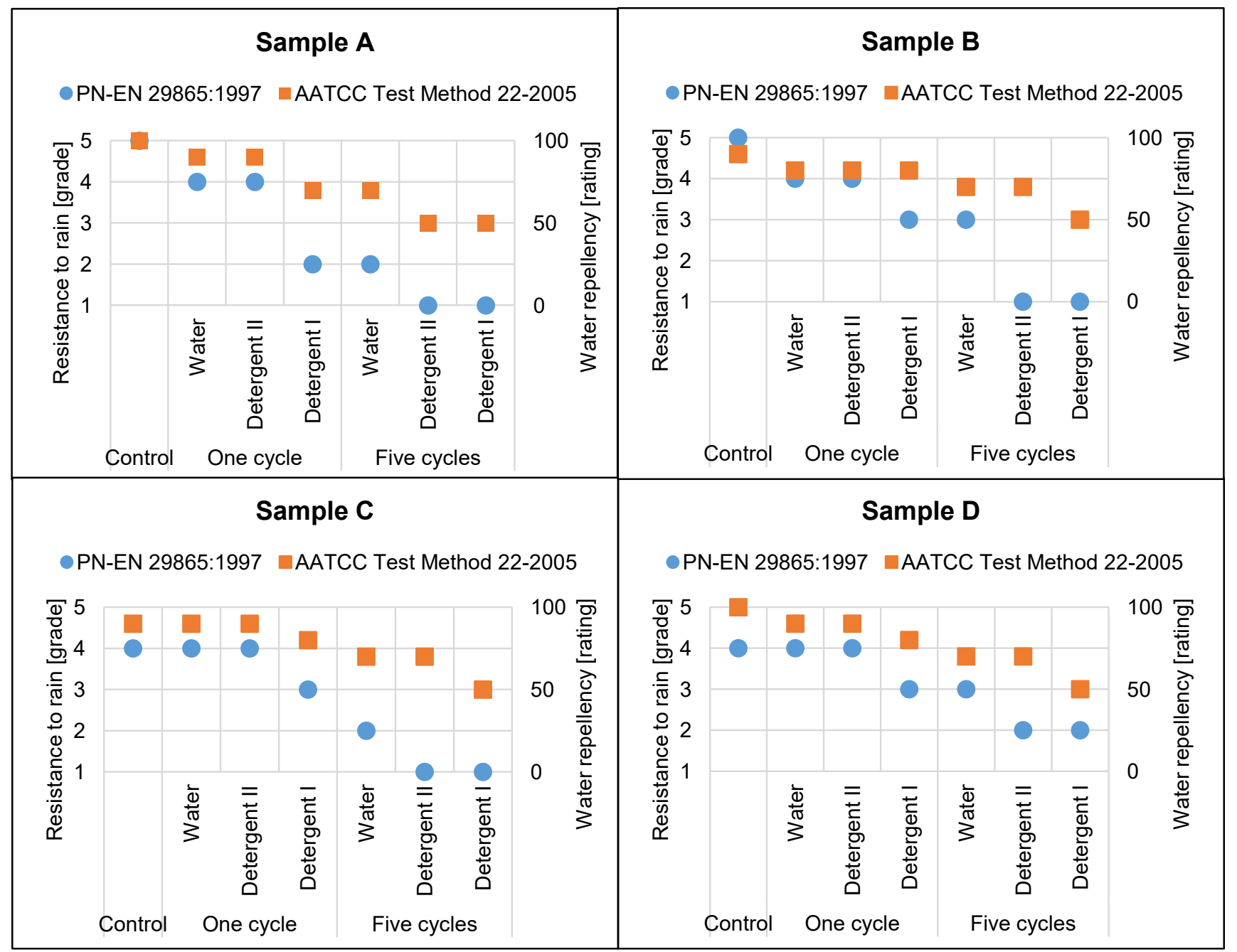

Figure 5. Effects of washing on hydrophobic finish of fabrics

other samples washed once with DETERGENT II, the values of waterproofing indicators were assessed at the same (sample B) or higher (samples A, C, and D) than those washed with DETERGENT I.

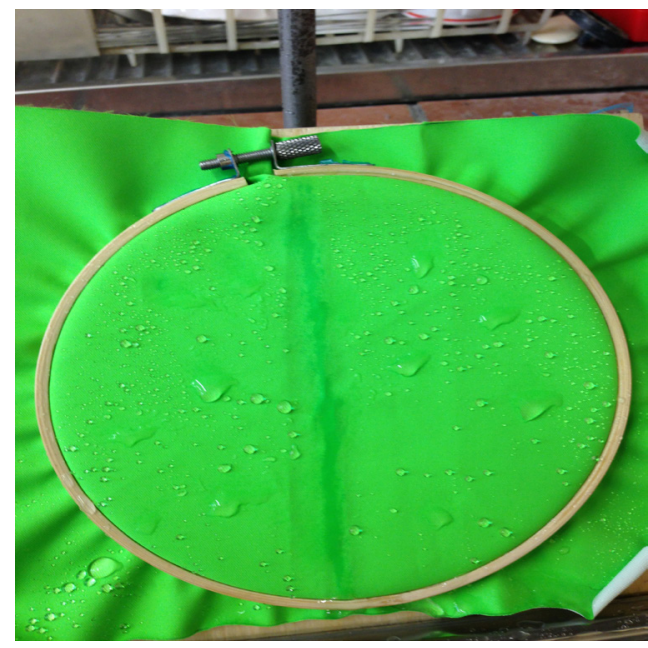

Figure 6. The surface of sample D washed once with DETERGENT II and after spray test
In most cases, some deterioration of hydrophobic properties was observed compared with unwashed samples. Sample $C$ gained the same grades as in initial determination; thus it has been concluded that the hydrophobicity of fabric was not deteriorated after washing with a special liquid detergent. For sample $D$, only one point reduction in the hydrophobic finish was observed (90). For samples $A$ and $B$, on each scale, the results lowered by one point were recorded (4 and 90; 4 and 80 , respectively). Compared with the previous determination after one wash cycle with a powder detergent (DETERGENT I), the coating still allowed water shed by forming it into small droplets, featuring an obtuse contact angle that allows quick water shedding and thus preventing surface wetting. The results of material surface evaluation after one wash cycle with DETERGENT II were comparable to those obtained for samples washed once without the use of washing agents. For all samples, water droplet pearling was still observed, thus indicating a small contact area between DWR coating and water droplet, and the contact angle was $90^{\circ}<\theta<150^{\circ}$.

After five wash cycles with an ordinary powder detergent (DETERGENT I), waterproofing properties disappeared completely in four of the tested laminates. Figure 7 shows the 

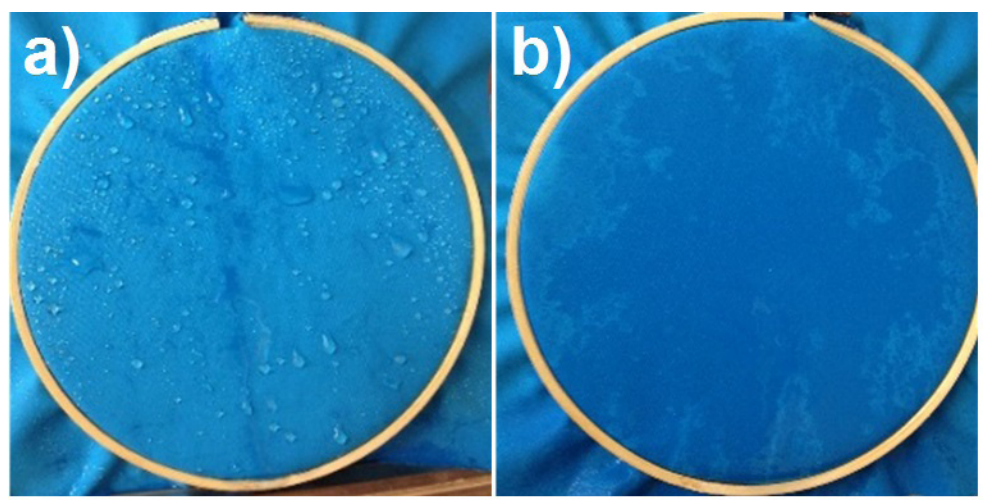

Figure 7. Sample $C$ after spray test, (a) washed once and (b) five times in DETERGENT I

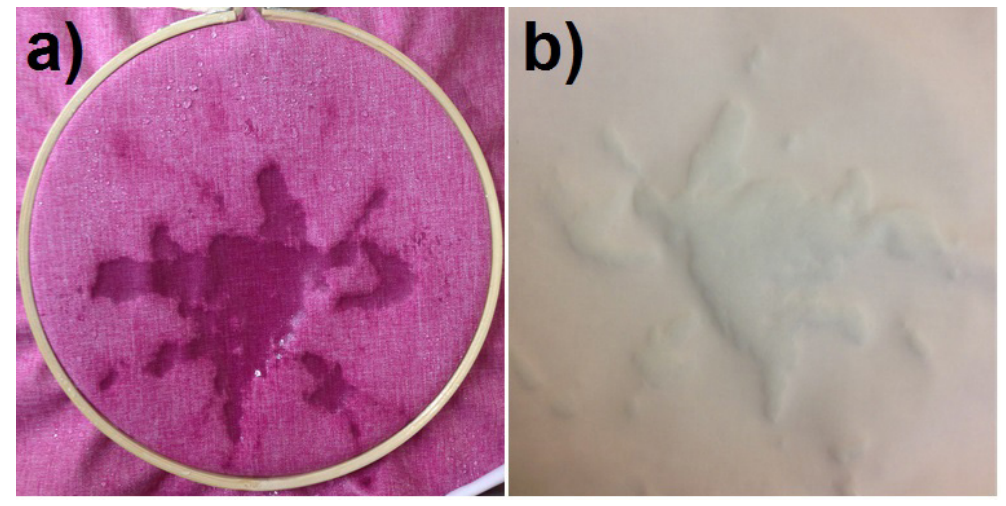

Figure 8. (a) Right-hand and (b) left-hand sides of sample B washed five times with DETERGENT II and subjected to spray test

appearance of the surface of sample $\mathrm{C}$ washed once (Fig. 7a) and five times (Fig. 7b) with DETERGENT I after spray test.

Samples $A, B$, and $C$ gained the lowest rating (grade 1) in terms of the hydrophobic finish because of the lack of visible water droplets on its surface, thus indicating that water was getting inside. Such phenomenon is characteristic of highenergy surfaces called hydrophilic (easily wettable) of the contact angle less than $90^{\circ}$.

As regards the degree of wetting, the samples under investigation showed a significant surface wetting, also beyond sprayed spots. Sample D, in accordance with EN 29865, was assigned a grade higher by one degree (grade 2) than other materials, because water droplets were visible on its surface and caused only partial wetting. The degree of wetting was assessed to be the same as for other samples, that is, 50 . Comparing test results after five wash cycles in both detergents, it is observed that there are only minute differences in material behavior as regards the degree of surface wetting for some samples washed with Detergent II.

Among tested samples washed five times in DETERGENT II, the samples $A$ and $B$ were the least affected; the degree of wetting was rated at the lowest level-50. For sample $B$, an additional phenomenon was observed, namely, a "pocket" in the material (Fig. 8a) where water droplets fell and this was visible also on reverse side (Fig. $8 b$ ).

Samples $C$ and $D$ demonstrated smaller wetting surface area after five wash cycles with DETERGENT II than that determined after the same number of wash cycles with an ordinary washing powder (DETERGENT I), and therefore, they were rated 70 . Among the three cases under consideration (Water or DETERGENT I or DETERGENT II), the samples washed five times without the use of detergents gained the highest ratings. On a waterproofing scale, samples $A$ and $C$ gained rating 2, while samples $B$ and $D$ were assigned 3 . These values were higher by one (samples A, C, and D) or two grades (sample B) compared to samples washed five times with a powder or special liquid. The degree of surface wetting all materials were rated 70 , that is, equally (samples $C$ and $D$ ) as after five wash cycles with DETERGENT II and one grade higher for samples $A$ and $B$.

\section{Conclusions}

Waterproofing properties of DWR-coated laminates becomes deteriorated in the maintenance process, while the extent of deterioration depends on the type of detergent used and the number of wash cycles. The use of a special detergent has an advantageous effect on maintaining hydrophobic properties of DWR coatings only after one wash cycle. This is evident by higher values of both analyzed parameters compared to those washed with an ordinary powder (DETERGENT I).

Multiple washing process contributes to the significant deterioration of material hydrophobicity, as indicated by forming water droplets spreading across the surface featuring a small contact angle and wetting a significant area of samples. Comparative study of samples subjected to multiple washing with water alone show that such changes are attributable to a large extent to detergents used. Slight differences observed in behavior during spray test on the surface of laminates washed five times with both detergents lead to the conclusion that the 
use of special agent for washing functional textile products does not guarantee that waterproofing properties of DWR coatings are maintained after multiple washing cycles.

Another important factor lowering the durability of DWR coatings is the condition of material surface. The spots, where even slight creases are present, are more susceptible to wetting, thus indicating that the integrity of DWR coating was disrupted.

All tested laminate samples are characterized of low resistance to washing processes. A significant deterioration of hydrophobic properties after only five wash cycles indicates poor quality of products available in the market and finished with DWR coatings, as declared by the manufacturer.

\section{Acknowledgments}

This publication was financed from the funds of the Ministry of Science and Higher Education granted to the Faculty of Commodity Science at the Cracow University of Economics for maintaining the research capacity and research projects implemented by young scientists and doctoral research fellows.

\section{References}

[1] AATCC Test Method 22-2005 Water Repellency: Spray Test.

[2] Bachurová M., Wiener J. (2013). Theoretical model of the influence of textile structure on wetting hysteresis. Fibres \& Textiles in Eastern Europe, vol. 21, no. 2 (98), 80-83.

[3] Bendkowska W. (2007). Textiles capable of self-cleaning (in Polish), Przegląd Włókienniczy - Włókno, Odzież, Skóra, no. 2, 54-58.

[4] Ceria A., Hauser P. J. (2010), Atmospheric plasma treatment to improve durability of a water and oil repellent finishing for acrylic fabrics. Surface \& Coatings Technology, vol. 204, issues 9-10, 1535-1541.
[5] Davis R., El-Shafei A., Hauser P. (2011). Use of atmospheric pressure plasma to confer durable water repellent functionality and antimicrobial functionality on cotton/polyester blend. Surface \& Coatings Technology, vol. 205, issue 20, 4791-4797.

[6] EN 29865. Textiles. Determination of water repellency of fabrics by the Bundesmann rain-shower test.

[7] Hu J. (Ed.). (2016). Active coatings for smart textiles. Woodhead Publishing (Cambridge).

[8] Mortazavi V., Khonsari M. M. (2017). On the degradation of superhydrophobic surfaces: a review. Wear, vol. 372-373, 145-157.

[9] Namligoz E. S., Bahtiyari M. I., Hosaf E., Coban S. (2009). Performance comparison of new (dendrimer, nanoproduct) and conventional water, oil and stain repellents. Fibres \& Textiles in Eastern Europe, vol. 17, no. 5 (76), 76-81.

[10] Pan N., Sun G. (Eds.). (2011). Functional textiles for improved performance, protection and health. Woodhead Publishing Limited (Cambridge).

[11] Paul R. (Ed.). (2015). Functional finishes for textiles: improving comfort, performance and protection. Woodhead Publishing Limited (Cambridge).

[12] Shishoo R. (Ed.). (2005). Textiles in sport. Woodhead Publishing Limited (Cambridge).

[13] Shishoo R. (Ed.). (2015). Textiles for sportswear. Woodhead Publishing Limited (Cambridge).

[14] Song G. (Ed.). (2010). Improving comfort in clothing. Woodhead Publishing Limited (Cambridge).

[15] Sterman S. (2014). User evaluation of the waterproof jacket. AUTEX Research Journal, vol. 14, no. 1, 7-13.

[16] Wong Y. W. H., Yuen C. W. M., Leung M. Y. S., Ku S. K. A., Lam H. L. I. (2006). Selected applications of nanotechnology in textiles. AUTEX Research Journal, vol. 6, no. 1, 1-8.

[17] Zhang, Y., Wang, J., He, Y., He, Y., Xu, B., Wei, S., \& Xiao, F. (2011). Solvothermal Synthesis of Nanoporous Polymer Chalk for Painting Superhydrophobic Surfaces Langmuir, 27 (20), 12585-590. 